\title{
Nucleus of Tractus Solitarius Astrocytes as Homeostatic Integrators
}

\author{
Christophe M. Lamy \\ Department of Cell Biology and Morphology, University of Lausanne, 1005 Lausanne, Switzerland \\ Review of McDougal et al.
}

Astrocytes, the main glial population in the CNS, have long been considered a type of support cells that ensure an adequate environment for the proper operation of neurons. In recent years, novel experimental approaches revealed that these cells play an active role in the regulation of neurophysiological functions (Hamilton and Attwell, 2010). For example, astrocytes help to fine-tune blood and energy supplies to the brain as neuronal activity fluctuates and engage in bidirectional interactions with neurons (Pellerin and Magistretti, 2011).

Communication between astrocytes and other cell types is mediated by activation of membrane receptors by a wide array of signals. The subsequent induction of $\mathrm{Ca}^{2+}$ transients induces release of intercellular mediators. Some of those, such as ATP, glutamate, and D-serine, act on neurons to modulate excitability and support some forms of synaptic plasticity (Hamilton and Attwell, 2010).

Astrocytes contact plasma via astrocytic endfeet that surround brain capillaries, as well as in a set of specialized brain structures devoid of blood-brain barrier, the sensory circumventricular organs (CVOs). This suggests that astrocytes could relay

Received Dec. 5, 2011; revised Jan. 10, 2012; accepted Jan. 11, 2012.

This work was supported by a grant from the Swiss National Science Foundation to Jean-Yves Chatton.

Correspondence should be addressed to Christophe M. Lamy, Department of Cell Biology and Morphology, University of Lausanne, Rue du Bugnon 9, 1005 Lausanne, Switzerland. E-mail: christophe.lamy@unil.ch.

DOI:10.1523/JNEUROSCI.6049-11.2012

Copyright $\odot 2012$ the authors $\quad 0270-6474 / 12 / 322579-03 \$ 15.00 / 0$ information about the internal milieu to neuronal networks. Indeed, recent data have confirmed that astrocytes monitor blood-born signals, such as electrolytes, metabolites, hormones, and cytokines, that reflect the homeostatic status of the body, and that they communicate changes in these parameters to neuronal centers that regulate vital body functions (Marty et al., 2005; Shimizu et al., 2007; Gourine et al., 2010).

The nucleus of tractus solitarius (NTS) is an important viscerosensory center in the dorsal brainstem. It collects information about the internal environment via vagal afferents and from the area postrema (AP), a CVO juxtaposed to the fourth ventricle. The NTS regulates body homeostasis by producing autonomic reflexes and modulating behavior. Interestingly, NTS has a distinctive astrocytic organization. It has a high density of classical GFAP-positive astrocytes as well as a more specialized population of glial cells that acts as a diffusion barrier at the AP/NTS border (Dallaporta et al., 2010). The reasons for this high astrocytic density in NTS are unknown. However, NTS astrocytes respond to peripheral chemical signals such as TNF $\alpha$ and thrombine (Hermann and Rogers, 2009; Hermann et al., 2009), and they participate in homeostatic responses such as thrombininduced gastric stasis and $\alpha 2$-adrenergic receptor-dependent cardiovascular responses (Bhuiyan et al., 2009; Hermann et al., 2009). A recent paper published by McDougal et al. (2011) in The Journal of Neuroscience showed that NTS astrocytes can be activated directly by vagal afferents through an atypical mechanism, suggesting that these cells could participate in the modulation of NTS integrative function in physiological conditions.

McDougal et al. (2011) used $\mathrm{Ca}^{2+}$ imaging to probe astrocyte activation by afferent stimulation of the tractus solitarius (TS) in horizontal slices of rat NTS. Astrocytes were identified as cells retaining red fluorescent marker sulforhodamine 101 (SR101). Astrocytes responded to TS stimulation with large $\mathrm{Ca}^{2+}$ transients that were blocked by tetrodotoxin, indicating that they resulted from fiber activation. This does not rule out the participation of second-order neurons, however.

Astrocytic response to TS stimulation was mediated by glutamate, as expected from the activation of vagal afferents to NTS, and depended on AMPA receptors (AMPARs), but not NMDA or group I metabotropic glutamate receptors. The presence of functional AMPARs on NTS astrocytes was confirmed by detection of AMPAR subunit GluR1 expression on GFAP-labeled astrocytic processes. Interestingly, the diffuse pattern of GluR1 staining suggests that a substantial part of these receptors are located on distal processes where astrocytes contact synapses rather than on proximal GFAP+ branches. Demonstration of a colocalization with markers present on distal processes such as glial glutamate transporters GLT-1 or GLAST would clarify this point.

AMPA-mediated $\mathrm{Ca}^{2+}$ transients depended both on extracellular $\mathrm{Ca}^{2+}$ and on 
internal stores, as shown by the effects of $\mathrm{Ca}^{2+}$-free medium and thapsigargin, respectively. A decrease in $\mathrm{Ca}^{2+}$ response produced by philanthotoxin (PhTx) indicated that $\mathrm{Ca}^{2+}$ influx was partly mediated by $\mathrm{Ca}^{2+}$-permeable AMPARs. The partial effect of PhTx suggests that other modes of $\mathrm{Ca}^{2+}$ entry coexist. This might include activation of the $\mathrm{Na}^{+} / \mathrm{Ca}^{2+} \mathrm{ex}^{-}$ changer in the reverse mode following $\mathrm{Na}^{+}$ influx through AMPARs (Verkhratsky et al., 2011). Activation of voltage-gated $\mathrm{Ca}^{2+}$ channels by cell depolarization or of purinergic P2X autoreceptors by ATP released from stimulated astrocytes could also contribute to the $\mathrm{Ca}^{2+}$ rise. The release of $\mathrm{Ca}^{2+}$ from internal stores by $\mathrm{Ca}^{2+}$-gated ryanodine receptors shown in this paper would serve as an amplification step to the AMPAdependent $\mathrm{Ca}^{2+}$ influx.

Astrocytes are a heterogeneous population (Matyash and Kettenmann, 2010). Those recorded by McDougal et al. (2011) displayed staining by GFAP, S100B, and SR101, which are typical markers of classical astrocytes. In cortical regions, classical astrocytes have also been defined by a linear current-voltage relationship, gapjunctional coupling, the presence of glutamate transporter currents, and the lack of AMPARs, whereas complex astrocytes have opposite characteristics (Matyash and Kettenmann, 2010). Brainstem astrocytes seem to follow the same classification scheme except that AMPARs are expressed by all subpopulations (Grass et al., 2004). It would be of great interest to have a more detailed characterization of TS-responsive astrocytes at electrophysiological and molecular levels and to compare them to other NTS astrocytes. Subspecialization of brainstem astrocytes has been shown to support different responses to homeostatic signals (Erlichman et al., 2004). Similarly, distinct subgroups of NTS astrocytes responding to different peripheral signals could participate in specific homeostatic pathways.

McDougal et al. (2011) focused on receptor-mediated astrocytic responses to glutamate that could potentially lead to modulation of neural circuits through the release of gliotransmitters (Hermann et al., 2009). However, glutamate from vagal afferents could also affect neuronal networks via activation of astrocytic metabolism by a mechanism similar to that of the astrocyte-to-neuron lactate shuttle (ANLS) (Pellerin and Magistretti, 2011). According to the ANLS theory, glutamate is taken up by astrocytes together with $\mathrm{Na}^{+}$, leading to an increase in intracellular $\mathrm{Na}^{+}$and to the subsequent activation of
$\mathrm{Na} / \mathrm{K}$ ATPase. The pump in turn stimulates astrocytic glycolysis, resulting in the production and release of lactate that is then used as an energy substrate by neurons. Recently, it appeared that lactate not only fuels neurons but could also increase their excitability by closing $\mathrm{K}_{\mathrm{ATP}}$ channels (Song and Routh, 2005; Parsons and Hirasawa, 2010). In agreement with this observation, ANLS-like mechanisms were shown to regulate homeostatic circuits such as those in the $\mathrm{Na}^{+}$-sensitive subfornical organ. In this CVO, astrocytes monitor systemic $\mathrm{Na}^{+}$levels and proportionally stimulate neighboring GABAergic interneurons by the release of lactate (Shimizu et al., 2007).

Lactate has also been shown to affect neuronal responses in regions regulating energy metabolism. In NTS, lactate blunted the response of glucose-sensing neurons to glucose and the counter-regulatory response to hypoglycemia (Himmi et al., 2001; Patil and Briski, 2005a,b). Similar effects were discovered in hypothalamic metabolic centers, such as ventromedial and lateral areas, in which lactate appeared to differentially regulate glucose-inhibited and glucose-exited cells (Song and Routh, 2005; Parsons and Hirasawa, 2010). This suggests a general role for lactate as an indicator of energy balance. Activation of lactate release by vagal afferents would thus be especially significant for the regulation of energy metabolism and feeding behavior by NTS. Because astrocytes potentially also respond to metabolism-related hormones, they could act as integrators of homeostatic signals driving NTS responses.

To summarize, the findings of $\mathrm{McDou}$ gal et al. (2011) confirm previous reports of astrocyte activation by vagal primary afferents (Ballanyi et al., 1993) and describe an uncommon mechanism leading to direct stimulation of $\mathrm{Ca}^{2+}$ responses by AMPARs. These experiments show that astrocytes in NTS are positioned to control the first relay in vago-vagal reflexes. Given that these astrocytes are also receptive to blood-born peripheral signals and can modulate neuronal networks through neuroactive mediators and neurometabolic coupling, they appear to be integrators of organism homeostatic state set to tune autonomic and behavioral adaptive responses to varying internal conditions. These astrocytes could also regulate the plasticity of NTS microcircuits, via mechanisms similar to those present in hypothalamus, by the release of gliotransmitters (Gordon et al., 2009), and through modifications of astrocytic synaptic coverage during homeostatic challenges (Oliet et al., 2006). It is also predictable that altera- tions of NTS astrocytic physiology will be involved in pathological conditions such as metabolic disorders (Yi et al., 2011). Further work is needed to better characterize NTS astrocyte subpopulations, to investigate the modulation of astrocytic function by chemical peripheral signals, and to clarify the interaction of these astrocytes with specific neuronal subcategories driving homeostatic responses.

\section{References}

Ballanyi K, Branchereau P, Champagnat J, Fortin G, Velluti J (1993) Extracellular potassium, glial and neuronal potentials in the solitary complex of rat brainstem slices. Brain Res 607:99-107.

Bhuiyan ME, Waki H, Gouraud SS, Takagishi M, Cui H, Yamazaki T, Kohsaka A, Maeda M (2009) Complex cardiovascular actions of alpha-adrenergic receptors expressed in the nucleus tractus solitarii of rats. Exp Physiol 94:773-784

Dallaporta M, Bonnet MS, Horner K, Trouslard J, Jean A, Troadec JD (2010) Glial cells of the nucleus tractus solitarius as partners of the dorsal hindbrain regulation of energy balance: a proposal for a working hypothesis. Brain Res 1350:35-42.

Erlichman JS, Cook A, Schwab MC, Budd TW, Leiter JC (2004) Heterogeneous patterns of $\mathrm{pH}$ regulation in glial cells in the dorsal and ventral medulla. Am J Physiol Regul Integr Comp Physiol 286:R289-R302.

Gordon GR, Iremonger KJ, Kantevari S, EllisDavies GC, MacVicar BA, Bains JS (2009) Astrocyte-mediated distributed plasticity at hypothalamic glutamate synapses. Neuron 64:391-403.

Gourine AV, Kasymov V, Marina N, Tang F, Figueiredo MF, Lane S, Teschemacher AG, Spyer KM, Deisseroth K, Kasparov S (2010) Astrocytes control breathing through $\mathrm{pH}$ dependent release of ATP. Science 329:571-575.

Grass D, Pawlowski PG, Hirrlinger J, Papadopoulos N, Richter DW, Kirchhoff F, Hülsmann S (2004) Diversity of functional astroglial properties in the respiratory network. J Neurosci 24:1358-1365.

Hamilton NB, Attwell D (2010) Do astrocytes really exocytose neurotransmitters? Nat Rev Neurosci 11:227-238.

Hermann GE, Rogers RC (2009) TNF activates astrocytes and catecholaminergic neurons in the solitary nucleus: implications for autonomic control. Brain Res 1273:72-82.

Hermann GE, Van Meter MJ, Rood JC, Rogers RC (2009) Proteinase-activated receptors in the nucleus of the solitary tract: evidence for glialneural interactions in autonomic control of the stomach. J Neurosci 29:9292-9300.

Himmi T, Perrin J, Dallaporta M, Orsini JC (2001) Effects of lactate on glucose-sensing neurons in the solitary tract nucleus. Physiol Behav 74:391-397.

Marty N, Dallaporta M, Foretz M, Emery M, Tarussio D, Bady I, Binnert C, Beermann F, Thorens B (2005) Regulation of glucagon secretion by glucose transporter type 2 (glut2) and astrocyte- 
dependent glucose sensors. J Clin Invest 115:3545-3553.

Matyash V, Kettenmann H (2010) Heterogeneity in astrocyte morphology and physiology. Brain Res Rev 63:2-10.

McDougal DH, Hermann GE, Rogers RC (2011) Vagal afferent stimulation activates astrocytes in the nucleus of the solitary tract via AMPA receptors: evidence of an atypical neural-glial interaction in the brainstem. J Neurosci 31:14037-14045.

Oliet SH, Panatier A, Piet R (2006) Functional neuronal-glial anatomical remodelling in the hypothalamus. Novartis Found Symp 276:238-248; discussion, 248-252, 275281.

Parsons MP, Hirasawa M (2010) ATP-sensitive potassium channel-mediated lactate effect on orexin neurons: implications for brain energetics during arousal. J Neurosci 30:8061-8070.

Patil GD, Briski KP (2005a) Lactate is a critical "sensed" variable in caudal hindbrain monitoring of CNS metabolic stasis. Am J Physiol Regul Integr Comp Physiol 289:R1777-R1786.

Patil GD, Briski KP (2005b) Transcriptional activation of nucleus tractus solitarii/area postrema catecholaminergic neurons by pharmacological inhibition of caudal hindbrain monocarboxylate transporter function. Neuroendocrinology 81:96-102.

Pellerin L, Magistretti PJ (2011) Sweet sixteen for ANLS. J Cereb Blood Flow Metab. Advance online publication. doi:10.1038/jcbfm.2011.149.

Shimizu H, Watanabe E, Hiyama TY, Nagakura A,
Fujikawa A, Okado H, Yanagawa Y, Obata K, Noda M (2007) Glial $\mathrm{Na}_{\mathrm{x}}$ channels control lactate signaling to neurons for brain $\left[\mathrm{Na}^{+}\right]$ sensing. Neuron 54:59-72.

Song Z, Routh VH (2005) Differential effects of glucose and lactate on glucosensing neurons in the ventromedial hypothalamic nucleus. Diabetes 54:15-22.

Verkhratsky A, Rodriguez JJ, Parpura V (2011) Calcium signalling in astroglia. Mol Cell Endocrinol. Advance online publication. doi: 10.1016/j.mce.2011.08.039.

Yi CX, Habegger KM, Chowen JA, Stern J, Tschöp MH (2011) A role for astrocytes in the central control of metabolism. Neuroendocrinology 93:143-149. 